\title{
Quantum diffusion in liquid para-hydrogen from ring-polymer molecular dynamics
}

\author{
Thomas F. Miller III and David E. Manolopoulos \\ Physical and Theoretical Chemistry Laboratory, Oxford University, South Parks Road, Oxford \\ OX1 3QZ, United Kingdom
}

(Received 13 December 2004; accepted 25 February 2005; published online 6 May 2005)

\begin{abstract}
We have used the ring-polymer molecular dynamics method to calculate approximate Kubo-transformed velocity autocorrelation functions and self-diffusion coefficients for low-pressure liquid para-hydrogen at temperatures of 25 and $14 \mathrm{~K}$. The resulting diffusion coefficients are shown to be consistent with experimental shear viscosities and the established finite-size relation $D(L)$ $\simeq D(\infty)-2.837 k_{B} T / 6 \pi \eta L$, where $k_{B}$ is the Boltzmann constant, $T$ the absolute temperature, $\eta$ the shear viscosity, and $L$ the length of the (cubic) simulation cell. The diffusion coefficients $D(L)$ obtained in simulations with finite system sizes are therefore too small. However, the extrapolation to infinite system size corrects this deficiency and leads to excellent agreement with experimental results. This both demonstrates the influence of system-size effects on quantum mechanical diffusion coefficients and provides further evidence that ring-polymer molecular dynamics is an accurate as well as practical way of including quantum effects in condensed phase molecular dynamics. (C) 2005 American Institute of Physics. [DOI: 10.1063/1.1893956]
\end{abstract}

\section{INTRODUCTION}

Liquid para-hydrogen provides an ideal testing ground for methods that include quantum mechanical effects in molecular dynamics simulations. While hydrogen is certainly a quantum liquid, it does not exhibit such pronounced identical particle exchange effects as liquid helium, and this significantly simplifies the calculations. The triple point temperature of hydrogen is $13.8 \mathrm{~K}$, and even at this temperature the thermal wavelength $\lambda=h /\left(2 \pi m k_{B} T\right)^{1 / 2} \simeq 3.3 \AA$ is only just larger than the hard-sphere diameter for the interaction between two hydrogen molecules $(\sigma \simeq 3.0 \AA)$. This implies that the exchange of identical para-hydrogen molecules will not have an enormous effect on the properties of the liquid phase, as was demonstrated some time ago for static equilibrium properties by path integral Monte Carlo calculations. ${ }^{1}$ Furthermore, since the critical point temperature $T_{c}$ $\simeq 33.1 \mathrm{~K}$ is substantially lower than the rotational temperature $\theta_{\text {rot }} \simeq 87.6 \mathrm{~K}$, the vast majority of para-hydrogen molecules will be in their ground rotational state in the liquid phase. Since $J=0$ rotational wave functions are spherically symmetric, this implies that the interaction between the molecules can be modeled to a good approximation by an isotropic pair potential, ${ }^{1}$ which once again simplifies the calculations.

As a result of these simplifications, the self-diffusion coefficient of liquid para-hydrogen has already been calculated using a wide variety of approximate quantum mechanical techniques. ${ }^{2-9}$ These range from more formal approaches such as maximum entropy analytic continuation of the imaginary-time propagator ${ }^{2}$ and quantum mode-coupling theory ${ }^{3}$ through to trajectory-based techniques such as centroid molecular dynamics, ${ }^{4-7}$ forward-backward semiclassical dynamics, ${ }^{8}$ and the classical Wigner model. ${ }^{9}$ However, because of the difficulty of performing condensed phase quantum dynamical calculations, all of these previous studies have considered rather small simulation cells (the largest to date consisting of 256 molecules $^{6}$ ), and none of them has investigated how the calculated diffusion coefficient scales with the system size.

The reason why this might be an important issue is that the self-diffusion coefficient of a purely classical liquid is known to increase quite significantly as the size of the simulation is increased. As was first shown by Dünweg and Kremer, ${ }^{10}$ and has recently been reiterated by Yeh and Hummer, ${ }^{11}$ the leading system-size dependence of the calculated diffusion coefficient will be given by the hydrodynamic equation

$$
D(L)=D(\infty)-\xi \frac{k_{B} T}{6 \pi \eta L},
$$

where $\eta$ is the shear viscosity, $L$ the length of the simulation cell, and $\xi$ a numerical coefficient that depends on the geometry of the simulation ${ }^{12}$ (with $\xi \simeq 2.837297$ for a cubic cell $^{12,13}$ ). As Yeh and Hummer have emphasized, ${ }^{11}$ the vast majority $(>80 \%)$ of the correction for finite-size effects in Eq. (1) comes from the fact that the average momentum of the atoms in the simulation must be set equal to zero to satisfy Newton's third law. This clearly restricts the motion of the atoms in a small simulation cell more so than in a large one, leading to an increase in the calculated diffusion coefficient with increasing system size. The key point we would add here is that there is no reason why the same argument, and indeed the entire hydrodynamic argument that leads to the more precise result in Eq. (1), ${ }^{10,11}$ should not also apply to a quantum simulation.

In order to explore this issue, we have performed some new calculations of the self-diffusion coefficient of liquid para-hydrogen using the approximate quantum mechanical 
ring-polymer molecular dynamics (RPMD) method. ${ }^{14}$ This is the first time that this method, which generalizes the exact path integral molecular dynamics technique for calculating static equilibrium properties, ${ }^{15}$ has been applied to a condensed phase dynamical problem. The equations that are needed to calculate the self-diffusion coefficient of liquid para-hydrogen using the method are therefore summarized in Sec. II. The great advantage of the RPMD approximation in comparison to alternative techniques is that it is simple enough to allow one to perform calculations on systems with a wide range of different sizes, even on a personal computer. We have therefore been able to study the system-size scaling of our computed diffusion coefficients and confirm that this scaling satisfies Eq. (1). The results of this investigation are discussed in Sec. III and summarized in Sec. IV.

\section{THEORY}

\section{A. Ring-polymer molecular dynamics}

The exact quantum mechanical self-diffusion coefficient of liquid para-hydrogen is given by a Green-Kubo relation in terms of the time integral of a Kubo-transformed velocity autocorrelation function: ${ }^{16}$

$$
D=\frac{1}{3} \int_{0}^{\infty} \widetilde{C}_{\mathbf{v} \cdot \mathbf{v}}(t) d t,
$$

where

$$
\begin{aligned}
\tilde{C}_{\mathbf{v} \cdot \mathbf{v}}(t)= & \frac{1}{Z \beta N m^{2}} \sum_{i=1}^{N} \int_{0}^{\beta} \operatorname{tr}\left[e^{-\lambda \hat{H}} \hat{\mathbf{p}}_{i} e^{-(\beta-\lambda) \hat{H}}\right. \\
& \left.\cdot e^{+i \hat{H} t / \hbar} \hat{\mathbf{p}}_{i} e^{-i \hat{H} t / \hbar}\right] d \lambda,
\end{aligned}
$$

with

$$
Z=\operatorname{tr}\left[e^{-\beta \hat{H}}\right]
$$

Here $\beta=1 /\left(k_{B} T\right)$ is the reciprocal temperature, $N$ the number of molecules in the system, $m$ their molecular mass, $\hat{H}$ the Hamiltonian operator for the entire system, and $\hat{\mathbf{p}}_{i}$ the centerof-mass momentum operator for molecule $i$.

Strictly speaking, since hydrogen molecules are composite bosons, the traces in Eqs. (3) and (4) should be evaluated in a basis of symmetrized coordinate eigenstates. ${ }^{17}$ However, as we have discussed in the introduction, boson exchange effects are unlikely to have a significant impact on the properties of liquid para-hydrogen. We shall therefore follow earlier treatments ${ }^{1-9}$ in ignoring these effects and regarding the para-hydrogen molecules as distinguishable particles.

Now the RPMD method provides an approximation to correlation functions involving configurational (positiondependent) operators, ${ }^{14}$ rather than those involving momentum operators such as $\widetilde{C}_{\mathbf{v} \cdot \mathbf{v}}(t)$. However, since the momentum operators $\hat{\mathbf{p}}_{i}$ in Eq. (3) are proportional to the Heisenberg time derivatives of position operators,

$$
\hat{\mathbf{p}}_{i}=m \frac{i}{\hbar}\left[\hat{H}, \hat{\mathbf{r}}_{i}\right]
$$

where $\hat{\mathbf{r}}_{i}$ is the center-of-mass position operator of molecule $i$, the exact velocity autocorrelation function $\widetilde{C}_{\mathbf{v} \cdot \mathbf{v}}(t)$ can be calculated equivalently as

$$
\tilde{C}_{\mathbf{v} \cdot \mathbf{v}}(t)=-\frac{d^{2}}{d t^{2}} \widetilde{C}_{\mathbf{r} \cdot \mathbf{r}}(t)
$$

where

$$
\tilde{C}_{\mathbf{r} \cdot \mathbf{r}}(t)=\frac{1}{Z \beta N} \sum_{i=1}^{N} \int_{0}^{\beta} \operatorname{tr}\left[e^{-\lambda \hat{H}} \hat{\mathbf{r}}_{i} e^{-(\beta-\lambda) \hat{H}} \cdot e^{+i \hat{H} t / \hbar} \hat{\mathbf{r}}_{i} e^{-i \hat{H} t / \hbar}\right] d \lambda
$$

is a Kubo-transformed position autocorrelation function. Since the correlated operators in this last equation are configurational, the RPMD method can be applied to $\widetilde{C}_{\mathbf{r} \cdot \mathbf{r}}(t)$, and the corresponding approximation to $\widetilde{C}_{\mathbf{v} \cdot \mathbf{v}}(t)$ then obtained from Eq. (6). (The same argument can also be used to apply the method to the wide variety of other correlation functions that involve Heisenberg time derivatives. It has recently been used, for example, in an application to the flux-side correlation functions that arise in the calculation of chemical reaction rates. ${ }^{18}$ )

In the absence of identical particle exchange effects, the RPMD approximation to $\widetilde{C}_{\mathbf{r} \cdot \mathbf{r}}(t)$ is simply ${ }^{14}$

$$
\begin{aligned}
\tilde{C}_{\mathbf{r} \cdot \mathbf{r}}(t) \simeq & \frac{1}{(2 \pi \hbar)^{3 N n} Z_{n}} \iint \Pi_{j=1}^{N} \Pi_{k=1}^{n} d \mathbf{p}_{j}^{(k)} \\
& \times d \mathbf{r}_{j}^{(k)} e^{-\beta_{n} H_{n}\left(\left\{\mathbf{p}_{j}^{(k)}\right\},\left\{\mathbf{r}_{j}^{(k)}\right\}\right)} \frac{1}{N} \sum_{i=1}^{N} \overline{\mathbf{r}}_{i}(0) \cdot \overline{\mathbf{r}}_{i}(t),
\end{aligned}
$$

where

$$
Z_{n}=\frac{1}{(2 \pi \hbar)^{3 N n}} \iint \Pi_{j=1}^{N} \Pi_{k=1}^{n} d \mathbf{p}_{j}^{(k)} d \mathbf{r}_{j}^{(k)} e^{-\beta_{n} H_{n}\left(\left\{\mathbf{p}_{j}^{(k)}\right\},\left\{\mathbf{r}_{j}^{(k)}\right\}\right)} .
$$

In these equations, $\beta_{n}=\beta / n$, and $H_{n}\left(\left\{\mathbf{p}_{j}^{(k)}\right\},\left\{\mathbf{r}_{j}^{(k)}\right\}\right)$ is the classical Hamiltonian of a system of $N$ harmonic ring $n$-polymers that interact through the physical interaction potential $V\left(\mathbf{r}_{1}, \ldots, \mathbf{r}_{N}\right)$,

$$
\begin{aligned}
H_{n}\left(\left\{\mathbf{p}_{j}^{(k)}\right\},\left\{\mathbf{r}_{j}^{(k)}\right\}\right)= & \sum_{j=1}^{N} \sum_{k=1}^{n}\left[\frac{\left(\mathbf{p}_{j}^{(k)}\right)^{2}}{2 m}+\frac{1}{2} m \omega_{n}^{2}\left(\mathbf{r}_{j}^{(k)}-\mathbf{r}_{j}^{(k-1)}\right)^{2}\right] \\
& +\sum_{k=1}^{n} V\left(\mathbf{r}_{1}^{(k)}, \ldots, \mathbf{r}_{N}^{(k)}\right),
\end{aligned}
$$

where $\omega_{n}=1 /\left(\beta_{n} \hbar\right)$ and $\mathbf{r}_{j}^{(0)} \equiv \mathbf{r}_{j}^{(n)}$. The classical equations of motion generated by this ring-polymer Hamiltonian are used to evolve the integration variables in Eq. (8) forwards in time,

$$
\dot{\mathbf{p}}_{j}^{(k)}=-m \omega_{n}^{2}\left(2 \mathbf{r}_{j}^{(k)}-\mathbf{r}_{j}^{(k-1)}-\mathbf{r}_{j}^{(k+1)}\right)-\frac{\partial V\left(\mathbf{r}_{1}^{(k)}, \ldots, \mathbf{r}_{N}^{(k)}\right)}{\partial \mathbf{r}_{j}^{(k)}},
$$




$$
\dot{\mathbf{r}}_{j}^{(k)}=\frac{\mathbf{p}_{j}^{(k)}}{m},
$$

and the position centroid $\overline{\mathbf{r}}_{i}(t)$ in Eq. (8) is obtained from an average over the beads of the $i$ th ring-polymer necklace at time $t$ :

$$
\overline{\mathbf{r}}_{i}(t)=\frac{1}{n_{k=1}^{n}} \sum_{i}^{(k)}(t)
$$

The corresponding approximation to the Kubotransformed velocity autocorrelation function $\widetilde{C}_{\mathbf{v} \cdot \mathbf{v}}(t)$ in Eq. (3) can now be obtained by differentiating Eq. (8) twice with respect to time [see Eq. (6)]. Exploiting the symmetries of the classical ring-polymer dynamics, ${ }^{14}$ we find that the result of these two differentiations can be rearranged to the form

$$
\begin{aligned}
\widetilde{C}_{\mathbf{v} \cdot \mathbf{v}}(t) \simeq & \frac{1}{(2 \pi \hbar)^{3 N n} Z_{n}} \iint \Pi_{j=1}^{N} \Pi_{k=1}^{n} d \mathbf{p}_{j}^{(k)} d \mathbf{r}_{j}^{(k)} \\
& \times e^{-\beta_{n} H_{n}\left(\left\{\mathbf{p}_{j}^{(k)}\right\},\left\{\mathbf{r}_{j}^{(k)}\right\}\right)} \frac{1}{N m^{2}} \sum_{i=1}^{N} \overline{\mathbf{p}}_{i}(0) \cdot \overline{\mathbf{p}}_{i}(t),
\end{aligned}
$$

where $\overline{\mathbf{p}}_{i}(t)$ is momentum centroid of the $i$ th ring polymer at time $t$ :

$$
\overline{\mathbf{p}}_{i}(t)=\frac{1}{n} \sum_{k=1}^{n} \mathbf{p}_{i}^{(k)}(t) .
$$

Notice in passing that Eq. (14) reduces correctly to a purely classical velocity autocorrelation function,

$$
\begin{aligned}
C_{\mathbf{v} \cdot \mathbf{v}}^{\mathrm{cl}}(t) \simeq & \frac{1}{(2 \pi \hbar)^{3 N} Z^{\mathrm{cl}}} \iint \Pi_{j=1}^{N} d \mathbf{p}_{j} d \mathbf{r}_{j} \\
& \times e^{-\beta H\left(\left\{\mathbf{p}_{j}\right\},\left\{\mathbf{r}_{j}\right\}\right)} \frac{1}{N m^{2}} \sum_{i=1}^{N} \mathbf{p}_{i}(0) \cdot \mathbf{p}_{i}(t),
\end{aligned}
$$

in the case of $n=1$ ring-polymer bead.

A general feature of the RPMD method is that the above equations will give the correct quantum mechanical correlation functions (for distinguishable particles) in the limit as $t \rightarrow 0 .{ }^{14}$ Indeed one can show in the present case that the first three time derivatives of the position autocorrelation function $\widetilde{C}_{\mathbf{r} \cdot \mathbf{r}}(t)$ in Eq. (8) will also be exact in this short-time limit. Since $\widetilde{C}_{\mathbf{r} \cdot \mathbf{r}}(t)$ is a real and even function of $t$, its first and third derivatives vanish as $t \rightarrow 0$, and it is easy to show from Eq. (14) that for any $n$,

$$
\lim _{t \rightarrow 0} \widetilde{C}_{\mathbf{v} \cdot \mathbf{v}}(t)=\frac{3}{\beta m},
$$

which coincides with the exact quantum mechanical result obtained from Eq. (3). Even the purely classical $(n=1)$ formula for the velocity autocorrelation function in Eq. (16) will therefore give the correct Kubo-transformed quantum

\begin{tabular}{|c|c|}
\hline$\alpha 1.713$ & $C_{6} 12.14$ \\
\hline$\beta 1.5671$ & $C_{8} 215.2$ \\
\hline$\gamma 0.00993$ & $C_{9} 143.1$ \\
\hline$r_{c} 8.32$ & $C_{10} 4813.9$ \\
\hline
\end{tabular}
result in the limit as $t \rightarrow 0$.

The theoretical situation for longer times $(t \gg 0)$ is of course less satisfactory, as it is for all other approximate quantum dynamical techniques. ${ }^{2-9}$ As we have stressed from the outset, ${ }^{14}$ the RPMD method simply provides a conve-
TABLE I. Parameters in the Silvera-Goldman pair potential (in atomic units).

nient model for the effect of an initial quantum Boltzmann distribution on the subsequent real-time dynamics. This model is consistent with the quantum mechanical equilibrium distribution, it respects all of the symmetry properties of the exact Kubo-transformed correlation function, and it can be shown to give the exact result in certain limiting cases (such as the classical limit, the limit of a harmonic potential, and in the limit as $t \rightarrow 0) .{ }^{14}$ However, it is still no more than a model, and one can only be confident that it will give a good approximation to the correct result for problems in which real-time quantum interference effects are rapidly quenched. ${ }^{14}$ Fortunately, this is likely to be the case for a wide variety of condensed phase problems, including the liquid para-hydrogen problem under investigation here.

\section{B. Computational details}

In order to describe the interaction between $J=0$ parahydrogen molecules, we have followed earlier work ${ }^{1-9}$ in using the isotropic part of the Silvera-Goldman pair potential $^{19}$

$$
V(r)=e^{\alpha-\beta r-\gamma r^{2}}-\left(\frac{C_{6}}{r^{6}}+\frac{C_{8}}{r^{8}}-\frac{C_{9}}{r^{9}}+\frac{C_{10}}{r^{10}}\right) f_{c}(r),
$$

where

$$
f_{c}(r)= \begin{cases}e^{-\left(r_{c} / r-1\right)^{2}}, & \text { if } r \leqslant r_{c} \\ 1, & \text { otherwise }\end{cases}
$$

with the parameters listed in Table I. The only component of Eq. (18) that does not have an obvious physical interpretation is the $C_{9} / r^{9}$ term, which is an effective two-body approximation to the three-body tripole-dipole dispersion interaction. ${ }^{19}$ Isothermal-isobaric path integral Monte Carlo (PIMC) calculations using this pair potential have been shown to give good agreement with experimental results for a variety of the static equilibrium properties of liquid para-hydrogen. ${ }^{1}$

In our RPMD simulations, we used the theoretical molar volumes obtained in this earlier PIMC study $\left(25.6 \mathrm{~cm}^{3} \mathrm{~mol}^{-1}\right.$ at $14 \mathrm{~K}$ and $31.7 \mathrm{~cm}^{3} \mathrm{~mol}^{-1}$ at $25 \mathrm{~K}$ ), which were calculated under conditions of approximately zero external pressure. ${ }^{1}$ For each of these two thermodynamic state points, we performed simulations with four different system sizes, containing 108, 256, 500, and 864 para-hydrogen molecules. Periodic boundary conditions were applied using the minimum image convention with the interaction between neighboring ring-polymers truncated at a centroid-to-centroid distance of 15 bohr. Tests with larger cutoff distances were found to give identical results to within the statistical error of the calculations. 

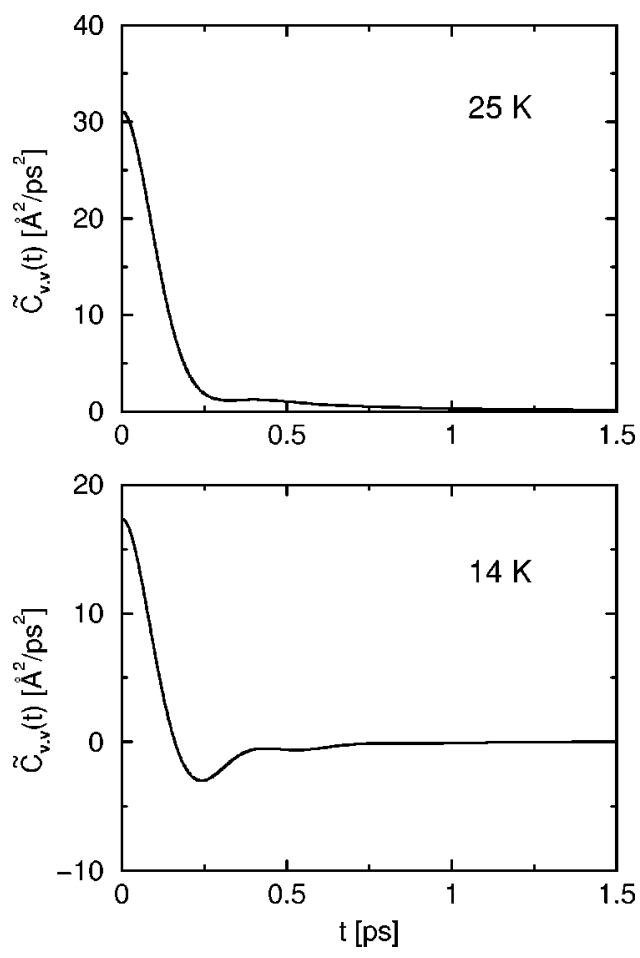

FIG. 1. Kubo-transformed velocity autocorrelation functions $\widetilde{C}_{\mathbf{v} \cdot \mathbf{v}}(t)$ for liquid para-hydrogen at the $T=25 \mathrm{~K}, V=31.7 \mathrm{~cm}^{3} \mathrm{~mol}^{-1}$ and $T=14 \mathrm{~K}, V$ $=25.6 \mathrm{~cm}^{3} \mathrm{~mol}^{-1}$ state points, as obtained from the present RPMD calculations with a system size of 864 molecules.

In each individual simulation, we equilibrated the system for $100 \mathrm{ps}$, and then calculated the velocity autocorrelation function in Eq. (14) for 2 ps by averaging over 100 consecutive 4 ps ring-polymer trajectories with a time step of $0.5 \mathrm{fs}$. The equations of motion in Eqs. (11) and (12) were integrated using a symplectic integrator based on alternating free harmonic ring-polymer and external force steps. The temperature was controlled by resampling the ring-polymer momenta from the Maxwell distribution at inverse temperature $\beta_{n}$ between each trajectory. This procedure was repeated five times to obtain an average value for (and a standard error in) the diffusion coefficient of each simulation.

As expected from the earlier PIMC calculations of static equilibrium properties, ${ }^{1}$ we found that $n=24$ ring-polymer beads sufficed to give converged results at $25 \mathrm{~K}$, and $n=48$ beads at $14 \mathrm{~K}$. For comparison with the classical limit, we also repeated each of our calculations using just $n=1$ ringpolymer bead. This led to an interesting insight concerning the difference between quantum and classical simulations at the same thermodynamic state point which we have already reported in a preliminary communication. ${ }^{20}$ We shall return to comment further on this matter in Sec. III C.

\section{RESULTS AND DISCUSSION}

\section{A. Velocity autocorrelation functions}

Our calculated Kubo-transformed velocity autocorrelation functions at the $T=25 \mathrm{~K}, V=31.7 \mathrm{~cm}^{3} \mathrm{~mol}^{-1}$ and $T$ $=14 \mathrm{~K}, V=25.6 \mathrm{~cm}^{3} \mathrm{~mol}^{-1}$ thermodynamic state points are presented in Fig. 1. The results shown in this figure were obtained from simulations of a periodically replicated system of 864 molecules ( $6^{3}$ face-centered cubic unit cells), and represent an average over 500 separate ring-polymer trajectories. The estimated statistical errors in the results are smaller than the widths of the plotted curves.

One sees from the figure that the calculated velocity autocorrelation functions behave much as one would expect for a subcritical fluid ( $25 \mathrm{~K}$; upper panel) and a low-temperature liquid (14 K; lower panel). In particular, there is a significant negative contribution to the autocorrelation function in the lower temperature (and higher density) simulation, which arises from the impulsive, velocity-reversing collisions that occur in this regime. A similar effect has been seen in earlier quantum mechanical simulations using a variety of different methods, ${ }^{2-9}$ and the RPMD Kubo-transformed correlation functions in Fig. 1 can be compared directly with those reported (for example) in Refs. 4 and 9.

The upshot of this comparison is that our Kubotransformed correlation functions agree somewhat better with those of the centroid molecular dynamics method ${ }^{4}$ than they do with those of the classical Wigner model. ${ }^{9}$ In particular, the small maximum in the velocity autocorrelation function at around $0.4 \mathrm{ps}$ in the upper panel of Fig. 1 is also present in Fig. 2 of Pavese and Voth, ${ }^{4}$ but it is absent from Fig. 5 of Poulsen et al. ${ }^{9}$ The RPMD and classical Wigner methods are therefore seen to give qualitatively different velocity autocorrelation functions at longer times. This simply highlights the fact that neither method (nor indeed any other method that neglects quantum mechanical interference effects in the real-time dynamics) is guaranteed to give an accurate result for $t>\beta \hbar(\simeq 0.3$ ps at $25 \mathrm{~K}) .^{21}$

The better agreement we obtain with the results of Pavese and Voth ${ }^{4}$ can be attributed to the fact that RPMD has more in common with centroid molecular dynamics than it does with the classical Wigner model. The ring-polymer and centroid molecular dynamics approximations both conserve the exact quantum mechanical equilibrium distribution, whereas the classical Wigner model involves purely classical trajectories that conserve the classical Boltzmann density $e^{-\beta H\left(\left\{\mathbf{p}_{j}\right\},\left\{\mathbf{r}_{j}\right\}\right)}$. As Voth and Hone have recently emphasized, ${ }^{22}$ this difference can be quite significant when the quantum and classical equilibrium distributions differ in a fundamental way, as is now known to be the case for liquid parahydrogen at both of the present thermodynamic state points. $^{20}$ It is therefore not surprising that our Kubotransformed velocity autocorrelation functions disagree at long times with those of the classical Wigner model.

\section{B. The average kinetic energy}

Since different approximations give different results, and an exact quantum dynamical treatment is currently not possible, we clearly need some sort of theoretical measure of the quality of the computed Kubo-transformed velocity autocorrelation functions in Fig. 1.

Such a measure has recently been devised by Poulsen et al., ${ }^{9}$ based on the observation that the standard velocity autocorrelation function 


$$
C_{\mathbf{v} \cdot \mathbf{v}}(t)=\frac{1}{Z N m^{2}} \sum_{i=1}^{N} \operatorname{tr}\left[e^{-\beta \hat{H}} \hat{\mathbf{p}}_{i} \cdot e^{+i \hat{H} t / \hbar} \hat{\mathbf{p}}_{i} e^{-i \hat{H} t / \hbar}\right]
$$

becomes proportional to the average value of the kinetic energy per molecule in the short-time limit:

$$
C_{\mathbf{v} \cdot \mathbf{v}}(0)=\frac{1}{Z N m^{2}} \sum_{i=1}^{N} \operatorname{tr}\left[e^{-\beta \hat{H}} \hat{\mathbf{p}}_{i} \cdot \hat{\mathbf{p}}_{i}\right] \equiv \frac{2}{m}\langle\mathrm{KE}\rangle .
$$

This implies a constraint on the Kubo-transformed velocity autocorrelation function by virtue of the relationship between the Fourier transforms of $C_{\mathbf{v} \cdot \mathbf{v}}(t)$ and $\widetilde{C}_{\mathbf{v} \cdot \mathbf{v}}(t)$. If $\widetilde{G}_{\mathbf{v} \cdot \mathbf{v}}(\omega)$ is the Fourier transform of $\widetilde{C}_{\mathbf{v} \cdot \mathbf{v}}(t)$,

$$
\tilde{G}_{\mathbf{v} \cdot \mathbf{v}}(\omega)=\int_{-\infty}^{\infty} e^{-i \omega t} \widetilde{C}_{\mathbf{v} \cdot \mathbf{v}}(t) d t
$$

and $G_{\mathbf{v} \cdot \mathbf{v}}(\omega)$ is that of $C_{\mathbf{v} \cdot \mathbf{v}}(t)$, then it is straightforward to show that

$$
G_{\mathbf{v} \cdot \mathbf{v}}(\omega)=\frac{\beta \hbar \omega}{\left(1-e^{-\beta \hbar \omega}\right)} \widetilde{G}_{\mathbf{v} \cdot \mathbf{v}}(\omega),
$$

and combining this with Eq. (21) gives

$$
\langle\mathrm{KE}\rangle_{K}=\frac{m}{4 \pi} \int_{-\infty}^{\infty} d \omega \int_{-\infty}^{\infty} d t \frac{\beta \hbar \omega}{\left(1-e^{-\beta \hbar \omega}\right)} e^{-i \omega t} \widetilde{C}_{\mathbf{v} \cdot \mathbf{v}}(t) .
$$

Hence the average kinetic energy per molecule depends on the behavior of the Kubo-transformed velocity autocorrelation function $\widetilde{C}_{\mathbf{v} \cdot \mathbf{v}}(t)$ at all times, and not just at $t=0$ where RPMD is already known to give the exact result in Eq. (17).

The utility of this constraint is that $\langle\mathrm{KE}\rangle$ is a static equilibrium property that can be computed exactly by path integral molecular dynamics (PIMD) ${ }^{15}$ Furthermore, since RPMD reduces to PIMD for static properties, ${ }^{14}$ we can calculate $\langle\mathrm{KE}\rangle$ exactly using our method at the same time as we calculate our approximation to $\widetilde{C}_{\mathbf{v} \cdot \mathbf{v}}(t)$. The most efficient statistical estimator for this purpose is the virial estimator, ${ }^{23}$

$$
\mathrm{KE}_{V}=\frac{3}{2 \beta}+\frac{1}{2 N n} \sum_{j=1}^{N} \sum_{k=1}^{n}\left(\mathbf{r}_{j}^{(k)}-\overline{\mathbf{r}}_{j}\right) \frac{\partial V\left(\mathbf{r}_{1}^{(k)}, \ldots, \mathbf{r}_{N}^{(k)}\right)}{\partial \mathbf{r}_{j}^{(k)}},
$$

which one simply time averages over each ring-polymer trajectory to obtain the average value $\langle\mathrm{KE}\rangle_{V}$; a comparison of this with $\langle\mathrm{KE}\rangle_{K}$ then provides a check on the accuracy of the approximate real-time RPMD dynamics. (The first term in Eq. (25) is the classical kinetic energy associated with the motion of the ring-polymer centroids $\left\{\overline{\mathbf{r}}_{j}\right\}$ and the second is the kinetic energy associated with quantum fluctuations; this second term vanishes in the free-particle limit but it can be quite substantial in a confined quantum liquid like parahydrogen.)

The results of this consistency check are shown in Table II, which compares the exact quantum mechanical kinetic energy $\langle\mathrm{KE}\rangle_{V}$ with the approximate RPMD kinetic energy $\langle\mathrm{KE}\rangle_{K}$ and the purely classical result $3 k_{\mathrm{B}} T / 2$ at both of the thermodynamic state points considered in Fig. 1. One sees from this table that the real-time ring-polymer dynamics does a remarkably good job of capturing the quantum me-

\begin{tabular}{|c|c|c|c|c|}
\hline \multirow[b]{2}{*}{$T(\mathrm{~K})$} & \multirow[b]{2}{*}{$N$} & \multicolumn{3}{|c|}{ Kinetic energy $(\mathrm{K})$} \\
\hline & & Exact [Eq. (25)] & RPMD [Eq. (24)] & Classical $\left[3 k_{B} T / 2\right]$ \\
\hline \multirow[t]{4}{*}{25} & 108 & 61.8 & 64.3 & 37.5 \\
\hline & 256 & 61.9 & 64.2 & \\
\hline & 500 & 61.9 & 64.4 & \\
\hline & 864 & 62.0 & 64.5 & \\
\hline \multirow[t]{4}{*}{14} & 108 & 63.6 & 67.7 & 21 \\
\hline & 256 & 63.7 & 67.6 & \\
\hline & 500 & 63.8 & 67.6 & \\
\hline & 864 & 63.8 & 67.7 & \\
\hline
\end{tabular}

TABLE II. Estimates of the average kinetic energy per molecule of liquid para-hydrogen at the $T=25 \mathrm{~K}, V=31.7 \mathrm{~cm}^{3} \mathrm{~mol}^{-1}$ and $T=14 \mathrm{~K}, V$ $=25.6 \mathrm{~cm}^{3} \mathrm{~mol}^{-1}$ state points, as a function of the number of molecules in the simulation. (The statistical errors in the exact and RPMD results are no larger than $0.1 \mathrm{~K}$.)

chanical contribution to the kinetic energy of liquid parahydrogen. In fact, it predicts a quantum contribution to the kinetic energy that only exceeds that of the virial estimator by about $10 \%$ (at both temperatures). Since both estimates were obtained from the same calculation, we believe that this $10 \%$ deviation from the exact result provides a reliable indication of the accuracy of the Kubo-transformed correlation functions in Fig. 1.

\section{Diffusion coefficients}

The average kinetic energy $\langle\mathrm{KE}\rangle_{K}$ in Eq. (24) is just one of many possible integral averages of a Kubo-transformed velocity autocorrelation function $\widetilde{C}_{\mathbf{v} \cdot \mathbf{v}}(t)$. The diffusion coefficient $D$ in Eq. (2) is another. Our calculated RPMD selfdiffusion coefficients at the 25 and $14 \mathrm{~K}$ thermodynamic state points are tabulated as a function of the number of molecules in the simulation in Table III.

It is clear from this table that, while the average kinetic energy is already well converged with a system size of 108 molecules (see Table II), the RPMD self-diffusion coefficients for liquid para-hydrogen increase quite significantly as the size of the simulation is increased. This behavior was anticipated in the introduction on the basis of the hydrodynamic formula in Eq. (1), which predicts that $D(N)$ will scale with the number of molecules $N$ in the simulation according to

TABLE III. Calculated self-diffusion coefficients of liquid para-hydrogen at the $T=25 \mathrm{~K}, V=31.7 \mathrm{~cm}^{3} \mathrm{~mol}^{-1}$ and $T=14 \mathrm{~K}, V=25.6 \mathrm{~cm}^{3} \mathrm{~mol}^{-1}$ state points, as a function of the number of molecules in the simulation. (The numbers in parentheses are the standard errors in the last digit from the RPMD calculations.)

\begin{tabular}{ccc}
\hline \hline & \multicolumn{2}{c}{$D\left(\AA^{2} \mathrm{ps}^{-1}\right)$} \\
\cline { 2 - 3 }$N$ & $25 \mathrm{~K}$ & $14 \mathrm{~K}$ \\
\hline \multirow{2}{*}{108} & $1.380(7)$ & $0.252(3)$ \\
256 & $1.433(7)$ & $0.270(3)$ \\
500 & $1.463(4)$ & $0.282(2)$ \\
864 & $1.483(3)$ & $0.288(2)$ \\
\hline \hline
\end{tabular}




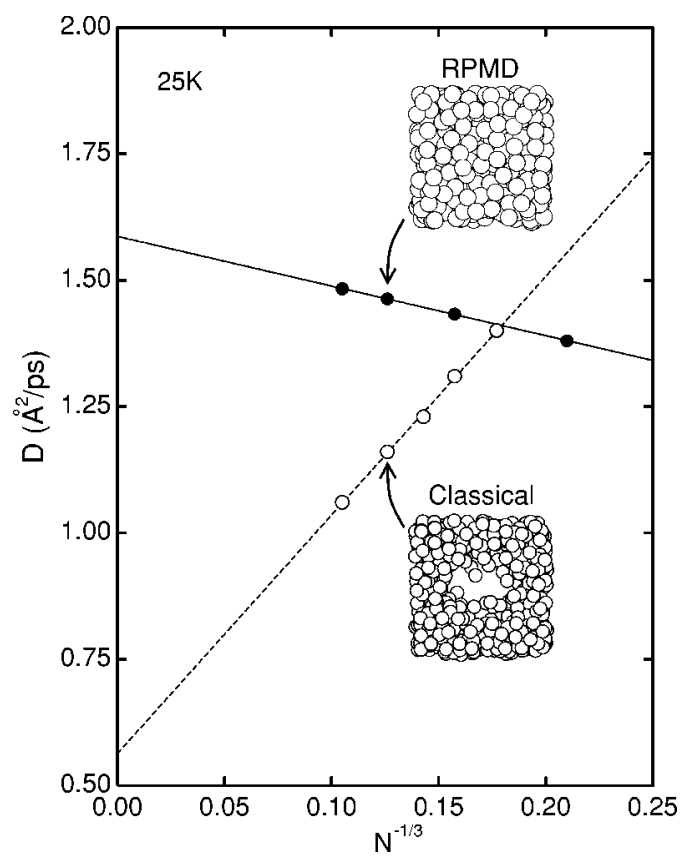

FIG. 2. System-size scaling of RPMD $(n=24)$ and classical $(n=1)$ selfdiffusion coefficients for liquid para-hydrogen at the $T=25 \mathrm{~K}, V$ $=31.7 \mathrm{~cm}^{3} \mathrm{~mol}^{-1}$ state point. The illustrations show typical configurations from the simulations of a system containing $N=500$ molecules.

$$
D(N)=D(\infty)-a N^{-1 / 3},
$$

where

$$
a=\frac{2.837 k_{B} T}{6 \pi \eta l},
$$

with $l=3.748 \AA \quad(3.515 \AA)$ for a molar volume of $31.7 \mathrm{~cm}^{3}\left(25.6 \mathrm{~cm}^{3}\right)$.

This prediction is confirmed in Figs. 2 and 3, which plot the calculated diffusion coefficients in Table III as a function of $N^{-1 / 3}$. Also included in these figures are the purely classical ( $n=1$ bead) results that we reported in our earlier communication. $^{20}$ The illustrations in the two figures show typical configurations from the RPMD and classical simulations of a system containing 500 molecules. In these illustrations, the radii of the classical particles have been set equal to the hard-sphere radius for the interaction between two para-hydrogen molecules $(\sigma / 2 \simeq 1.5 \AA)$. The radii of the quantum particles have been "swollen" from this by an amount equal to the average radius of gyration of the ring polymers, which was calculated from the statistical estimator

$$
r_{G}^{2}=\frac{1}{N n} \sum_{j=1}^{N} \sum_{k=1}^{n}\left|\mathbf{r}_{j}^{(k)}-\overline{\mathbf{r}}_{j}\right|^{2}
$$

and found be $r_{G} \simeq 0.47 \AA$ at $25 \mathrm{~K}$ and $0.56 \AA$ at $14 \mathrm{~K}$. For comparison with these values, the free harmonic ring $n$-polymer result

$$
r_{G}^{2}(\text { free })=\frac{\beta \hbar^{2}}{4 m}\left(1-\frac{1}{n^{2}}\right)
$$

gives $r_{G} \simeq 0.49 \AA$ at $25 \mathrm{~K}$ and $0.66 \AA$ at $14 \mathrm{~K}$. The compression of the radius of gyration due to intermolecular interactions was therefore found (as expected) to be larger in the

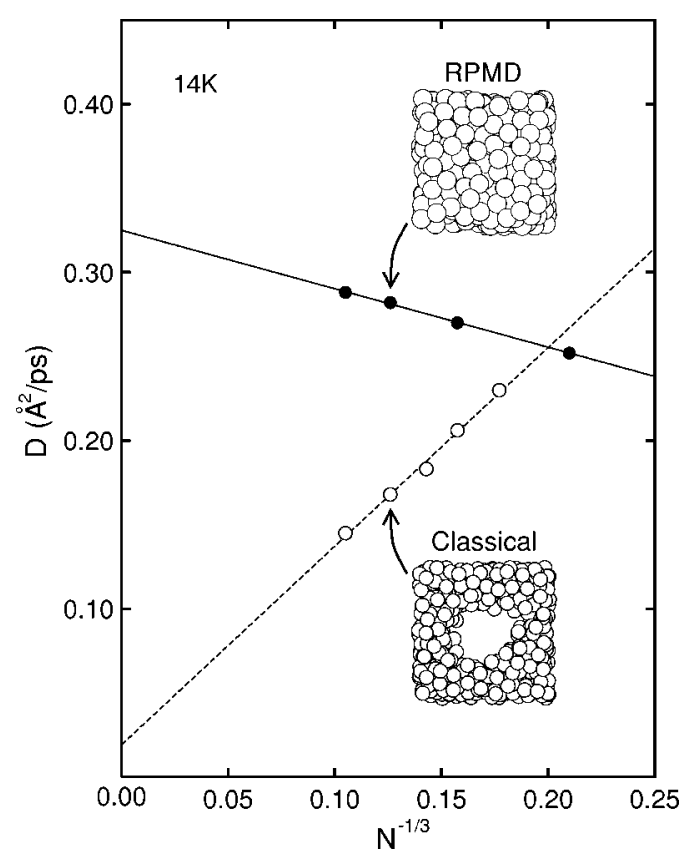

FIG. 3. System-size scaling of RPMD $(n=48)$ and classical $(n=1)$ selfdiffusion coefficients for liquid para-hydrogen at the $T=14 \mathrm{~K}, V$ $=25.6 \mathrm{~cm}^{3} \mathrm{~mol}^{-1}$ state point. The illustrations show typical configurations from the simulations of a system containing $N=500$ molecules.

dense quantum liquid than in the subcritical fluid.

Although both the classical and RPMD diffusion coefficients in Figs. 2 and 3 vary linearly with $N^{-1 / 3}$, they do so for entirely different reasons. The primary reason for the $N^{-1 / 3}$ scaling of the classical diffusion coefficients is that the present thermodynamic state points lie in the liquid-vapor coexistence region of the classical phase diagram. ${ }^{20}$ This leads to the spontaneous formation of bubbles in the classical simulations, such as those we have illustrated in Figs. 2 and 3 . The observed scaling arises because the fraction of molecules at the surface of a bubble is proportional to $N^{-1 / 3}$, where $N$ is the total number of molecules in the simulation. Since the surface of the bubble has a lower density than the bulk liquid, the molecules at the surface diffuse more rapidly, which explains why the classical diffusion coefficient decreases with an increase in the system size. ${ }^{20}$

By contrast, we believe that the origin of the $N^{-1 / 3}$ scaling of the RPMD results is the restricted reciprocal space of a finite-sized simulation, which leads to the formula for the diffusion coefficient in Eq. (1). ${ }^{10,11}$ In order to confirm this interpretation, we have used Eq. (27) to calculate shear viscosities from the linear fits to the RPMD data in Figs. 2 and 3. The resulting viscosities are $\eta=1.39 \times 10^{-5} \mathrm{~N} \mathrm{~s} \mathrm{~m}^{-2}$ at the $25 \mathrm{~K}$ state point and $\eta=2.38 \times 10^{-5} \mathrm{~N} \mathrm{~s} \mathrm{~m}^{-2}$ at $14 \mathrm{~K}$. These results agree sufficiently well with the experimental shear viscosities of liquid para-hydrogen at saturated vapor pressure $^{24}\left(0.94 \times 10^{-5} \mathrm{~N} \mathrm{~s} \mathrm{~m}^{-2}\right.$ at $25 \mathrm{~K}$ and 2.51 $\times 10^{-5} \mathrm{~N} \mathrm{~s} \mathrm{~m}^{-2}$ at $14 \mathrm{~K}$ ) to convince us that Eq. (1) accounts for the majority (if not all) of the system-size dependence of the RPMD calculations. In this case the calculated diffusion coefficient increases with an increase in the system size because more reciprocal space points are available to a larger simulation.

Finally, we note that the RPMD diffusion coefficients 
TABLE IV. Calculated and experimental self-diffusion coefficients for lowpressure liquid para-hydrogen at 25 and $14 \mathrm{~K}$. (The numbers in parentheses are the estimated errors in the last digit in cases where these are available.)

\begin{tabular}{cccc}
\hline \hline & & \multicolumn{2}{c}{$D\left(\AA^{2} \mathrm{ps}^{-1}\right)$} \\
\cline { 3 - 4 } Method & $N$ & $25 \mathrm{~K}$ & $14 \mathrm{~K}$ \\
\hline MEAC $^{\mathrm{a}}$ & 108 & 1.47 & 0.28 \\
$\mathrm{QMCT}^{\mathrm{b}}$ & 108 & 1.69 & 0.30 \\
$\mathrm{FBSD}^{\mathrm{c}}$ & 108 & $1.68(5)$ & $0.75(7)$ \\
$\mathrm{CWM}^{\mathrm{d}}$ & 125 & 1.73 & $\ldots$ \\
$\mathrm{CMD}^{\mathrm{e}}$ & 180 & $1.52(8)$ & $0.35(5)$ \\
RPMD $^{\mathrm{f}}$ & $\infty$ & $1.59(1)$ & $0.33(1)$ \\
Expt. $^{\mathrm{g}}$ & & 1.6 & 0.4 \\
\hline \hline
\end{tabular}

${ }^{\mathrm{a}}$ Maximum entropy analytic continuation (Ref. 2).

${ }^{\mathrm{b}}$ Quantum mode-coupling theory (Ref. 3).

${ }^{\mathrm{c}}$ Forward-backward semiclassical dynamics (Ref. 8).

${ }^{\mathrm{d}}$ Classical Wigner model (Ref. 9).

${ }^{\mathrm{e}}$ Centroid molecular dynamics (Ref. 7).

${ }^{\mathrm{f}}$ Extrapolated values from Figs. 2 and 3 (present work).

${ }^{\mathrm{g}}$ Experimental results from Ref. 25.

extrapolated to infinite system size in Figs. 2 and 3 agree rather well with experimental data. We obtain $D(\infty)$ $=1.59 \AA^{2} \mathrm{ps}^{-1}$ at the $25 \mathrm{~K}$ state point and $0.33 \AA^{2} \mathrm{ps}^{-1}$ at 14 $\mathrm{K}$. For comparison, the experimental self-diffusion coefficients of liquid para-hydrogen at saturated vapor pressure are $1.6 \AA^{2} \mathrm{ps}^{-1}$ at $25 \mathrm{~K}$ and $0.4 \AA^{2} \mathrm{ps}^{-1}$ at $14 \mathrm{~K}$. ${ }^{25}$ These results are collected together with those from a number of earlier theoretical studies of liquid para-hydrogen in Table IV.

\section{SUMMARY}

In this paper, we have used the approximate quantum mechanical RPMD method to calculate Kubo-transformed velocity autocorrelation functions and self-diffusion coefficients for low-pressure liquid para-hydrogen at temperatures of 25 and $14 \mathrm{~K}$. This is the first time that this method, which generalizes the exact PIMD technique for calculating static equilibrium properties, has been applied to a condensed phase dynamical problem.

The accuracy of the resulting velocity autocorrelation functions has been tested by using them to calculate the average kinetic energy of liquid para-hydrogen, and comparing the results with those obtained from exact PIMD calculations. The real-time RPMD approximation was found in this way to overestimate the quantum mechanical contribution to the kinetic energy at each temperature by about $10 \%$. This is our best estimate of the accuracy of the RPMD approximation for the present problem.

We have also studied the system-size scaling of our calculated self-diffusion coefficients and confirmed that this scaling satisfies the hydrodynamic relation in Eq. (1). To the best of our knowledge, this is the first time that the systemsize scaling of quantum diffusion coefficients has been investigated. It would be interesting to see whether the same scal- ing is obtained using the other approximate quantum mechanical techniques that have previously been applied to liquid para-hydrogen. ${ }^{2-9}$

The RPMD diffusion coefficients obtained in simulations with finite system sizes are somewhat smaller than those predicted by these alternative techniques. However, when the scaling implied by Eq. (1) is used to extrapolate our calculated diffusion coefficients to infinite system size, we obtain remarkably good agreement with experimental data (see Table IV). Since this is a problem for which purely classical molecular dynamics fails completely (see Figs. 2 and 3), we find this result to be rather encouraging.

It will be interesting in future work to see whether the RPMD approximation does equally well for various other dynamical properties of liquid para-hydrogen, such as more general transport coefficients ${ }^{6}$ and van Hove correlation functions. ${ }^{3,7}$ It will also be interesting to extend the method to include identical particle (boson) exchange effects, so that it can be applied to liquid ${ }^{4} \mathrm{He}$.

\section{ACKNOWLEDGMENTS}

The authors would like to thank Paul Madden for stimulating discussions, David Chandler for helpful comments on an early draft of this manuscript, and Joel Bowman for providing them with computer time on a linux cluster for their largest (864 molecule) RPMD calculations.

${ }^{1}$ D. Scharf, G. J. Martyna, and M. L. Klein, Low Temp. Phys. 19, 364 (1993).

${ }^{2}$ E. Rabani, D. R. Reichman, G. Krilov, and B. J. Berne, Proc. Natl. Acad. Sci. U.S.A. 99, 1129 (2002)

${ }^{3}$ D. R. Reichman and E. Rabani, J. Chem. Phys. 116, 6279 (2002).

${ }^{4}$ M. Pavese and G. A. Voth, Chem. Phys. Lett. 249, 231 (1996).

${ }^{5}$ G. J. Martyna, A. Hughes, and M. E. Tuckerman, J. Chem. Phys. 110, 3275 (1999)

${ }^{6}$ Y. Yonetani and K. Kinugawa, J. Chem. Phys. 119, 9651 (2003).

${ }^{7}$ T. D. Hone and G. A. Voth, J. Chem. Phys. 121, 6412 (2004).

${ }^{8}$ A. Nakayama and N. Makri, J. Chem. Phys. 119, 8592 (2003).

${ }^{9}$ J. A. Poulsen, G. Nyman, and P. J. Rossky, J. Phys. Chem. B 108, 19799 (2004).

${ }^{10}$ B. Dünweg and K. Kremer, J. Chem. Phys. 99, 6983 (1993).

${ }^{11}$ I-C. Yeh and G. Hummer, J. Phys. Chem. B 108, 15873 (2004).

${ }^{12}$ H. Hasimoto, J. Fluid Mech. 5, 317 (1959).

${ }^{13}$ G. Placzek, B. R. A. Nijboer, and L. van Hove, Phys. Rev. 82, 392 (1951).

${ }^{14}$ I. R. Craig and D. E. Manolopoulos, J. Chem. Phys. 121, 3368 (2004).

${ }^{15}$ M. Parrinello and A. Rahman, J. Chem. Phys. 80, 860 (1984).

${ }^{16}$ R. Kubo, M. Toda, and N. Hashitsume, Statistical Physics II: Nonequilibrium Statistical Mechanics (Springer, New York, 1985).

${ }^{17}$ R. P. Feynman and A. R. Hibbs, Quantum Mechanics and Path Integrals (McGraw-Hill, New York, 1965).

${ }^{18}$ I. R. Craig and D. E. Manolopoulos, J. Chem. Phys. 122, 084106 (2005).

${ }^{19}$ I. F. Silvera and V. V. Goldman, J. Chem. Phys. 69, 4209 (1978).

${ }^{20}$ T. F. Miller III, D. E. Manolopoulos, P. A. Madden, M. Konieczny, and H. Oberhofer, J. Chem. Phys. 122, 057101 (2005).

${ }^{21}$ W. H. Miller, J. Phys. Chem. A 105, 2942 (2001).

${ }^{22}$ G. A. Voth and T. D. Hone, J. Chem. Phys. 122, 057102 (2005)

${ }^{23}$ M. F. Herman, E. J. Bruskin, and B. J. Berne, J. Chem. Phys. 76, 5150 (1982).

${ }^{24}$ D. E. Diller, J. Chem. Phys. 42, 2089 (1965)

${ }^{25}$ B. N. Esel'son, Y. P. Blagoi, V. V. Grigor'ev, V. G. Manzhelii, S. A. Mikhailenko, and N. P. Neklyudov, Properties of Liquid and Solid Hydrogen (Israel Program for Scientific Translations, Jerusalem, 1971). 